\title{
3D VIDEO STREAMING FOR VIRTUAL EXPLORATION OF PLANET SURFACE
}

\author{
Himanshu S Mazumdar' ${ }^{1}$, Divya J Nimavat ${ }^{2}$, Marichi Patel ${ }^{3}$ \\ ${ }^{1}$ Head, Research and Development Center, Dharmsinh Desai University, Nadiad, Gujarat, India \\ ${ }^{2}$ Student, Department of Information Technology, Dharmsinh Desai University, Nadiad, Gujarat, India \\ ${ }^{3}$ Research Scholar, Research and Development Center, Dharmsinh Desai University, Nadiad, Gujarat, India
}

\begin{abstract}
Human perception is far superior then vision analysis by computer. The visual objects like distant planet surface (terrain) are not accessible to observer. This could be presented to human analyst in most natural representation like virtual 3D anaglyph for browsing and visual analysis. The paper represents development of a stereoscopic $3 D$ viewer application for planetary surface. For $3 D$ stereo visualization of virtual 3D geo-based data model, Anaglyph Method is most cost-effective as compared to other method like paired epipolar images, Multi-view display, integral imaging display. 3D anaglyph scene generated using Digital Elevation Map (DEM) and satellite imagery data sets for this study. The objective is to use the planetary optical remote sensing data which is available in the public domain database to create an application to study the formation of specific planet surface. Implementation for $3 D$ animated anaglyph DirectX library is used. 3D Terrain generation and rendering is vastly used in computer games, but here we use $3 D$ terrain generation for scientific use like, studies of relative and absolute surface (age), Analysis of Planetary Surface and its formation, Analysis of specific crater for scientific purpose, for safe landing site identification, Mineralogical mapping of planet, Ejecta mapping.
\end{abstract}

Keywords: Virtual Reality, Stereoscopy, DEM, 3D anaglyph, anaglyph map, DirectX

\section{INTRODUCTION}

The use of 3D visualization technique in planetary exploration in order is to display planet data in such a way that it is useful for analyze and browse the important parameter of the planet.

The 3D visualization can be developed using either DirectX Graphics or OpenGL Graphics which provide a light buffer to shield the developer from immediate effects of technical changes.

Both provide developers with direct access to any installed high-performance PC hardware but DirectX, which is most frequently updated (with eight versions having been released since its introduction in 1995) and offers a wider range of functions than OpenGL, is possibly the more widely used in the games environment.

DirectX is a fast device-independent means of accessing any multimedia hardware in the Windows operating system environment. Its each component APIs each control a set of low-level functions, that access the hardware or enable software emulation of a feature not provided by the hardware, and include support for 3D graphics acceleration as well as control over numerous input devices such as keyboards, mice and joysticks. DirectX therefore have the ability to utilize more efficiently multimedia and graphics accelerator features such as advanced sound cards and 3D acceleration cards.
DirectX architecture was therefore chosen to support the development undertaken during this project. However, DirectX's software layer that allows communication with any type of graphics card is unnecessarily complex for the number of features required in this design and therefore a simpler form of access to Direct3D/DirectX Graphics has been written into the program.

The implementation is done using Direct3D. OpenGL works on any OS where an OpenGL driver has been written. Even though Direct3D is not cross-platform graphics API but it has advantage over OpenGL. OpenGL is written to a specification maintained by the OpenGL Architecture Review Board. That means the API is slow to adopt new features on graphics cards, and not all graphical effects are supported on all cards. Some game consoles also support the use of Direct3D, which is released more often than OpenGL and contains a specific set of functionality which driver developers must include.

In the present project, it is proposed to work on Martian surface map where satellite-derived high resolution DEMs, generated by optical stereoscopy. This Martian database is downloaded from public domain database which is taken from Mars Global Surveyor (MGS) mission and MOLA data. 


\subsection{Digital Elevation Model (DEM)}

A DEM can be symbolized by a raster (a grid of squares) or by a triangular irregular network. Frequently DEMs are constructed using remote sensing techniques, but, they may also be made from land survey. Geographic Information Systems use DEMs quite often and are the most regularly used for digitally creating relief maps.

There are a lots of different standards, how to store elevation data. The used formats are Digital Elevation Models that use two-dimensional arrays of height values. These DEMs also contain a latitude/longitude position and data resolution $(10 \mathrm{~m}$, $1 \mathrm{~km}$...). Satellite elevation data are most often stored as USGS-DEM files (.dem). In games grayscale images (height maps) are a common solution to specify terrain data.

\subsection{Anaglyph Method}

Anaglyph 3D is the name given to the stereoscopic 3D effect achieved by means of encoding each eye's image using filters of different (usually chromatically opposite) colors, typically red and cyan.

Anaglyph is a type of stereo 3D image created from two photographs taken approximately 2.5 inches apart, the center distance typically between human eyes. The Red color field of the left photo is combined with that of the right photo in such a way as to create the illusion of depth. When viewed through the "color-coded" "anaglyph glasses", each of the two images reaches corresponding eye, revealing an integrated stereoscopic image.

\section{PROPOSED ALGORITHM}

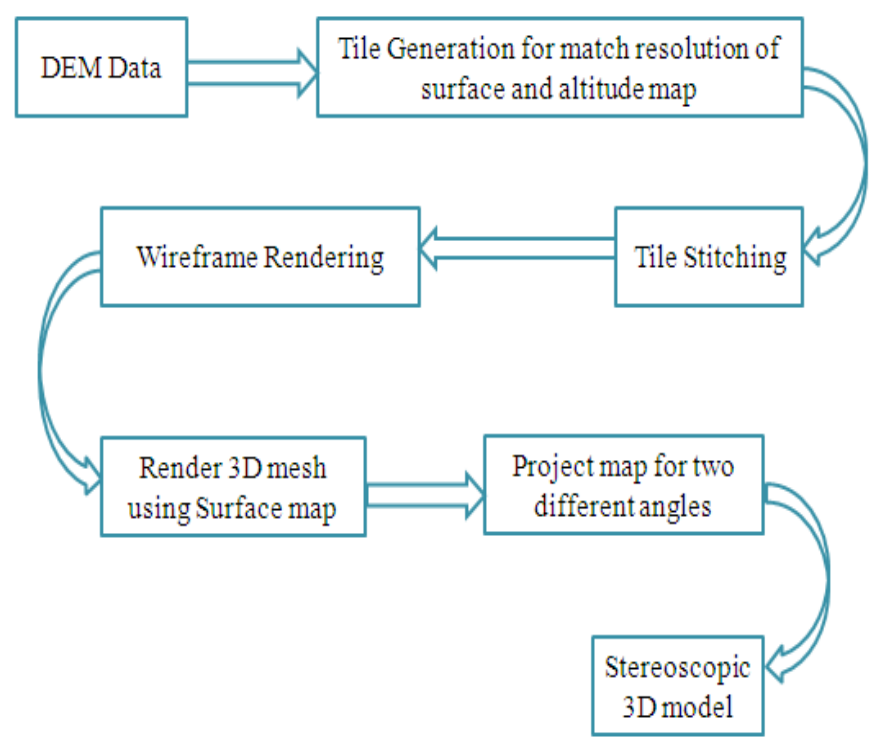

Fig 1: Shows the steps for creating animated anaglyph 3D model algorithm.

\subsection{Tiles Generation for Match Resolution of Surface}

\section{and Altitude Map}

To generate 3D planet surface we have used the mars surface database which is available from public domain. The format for altitude data is MOLA (Mars Orbiter Laser Altimeter) taken from open database of Arizona University. The total resolution is $46080 \times 23040$ pixels. In the database there are 72 tiles. Each tiles having the resolution of $7680 \times 7680$ pixels. And the other surface data of mars is taken from Celestia open domain database. It has resolution of each tile is 512 x 512 pixels. Now to match both the database resolution to create actual 3D surface of the planet, Elevation map and surface map is expanded using interpolation algorithm to match the resolution. The interpolated elevation map and surface map is tiled of size $512 \times 512$ in 8192 segments each.

\subsection{Tiles Stitching}

Stitching is necessary when the adjacent tiles have a different level of detail otherwise gaps in the terrain may be visible otherwise. Adjacent tiles can have a different resolution which causes visual inconsistencies at the joints, to avoid this we use a stitching process for border rows and columns to avoid this. Each tile stitches with its northern and eastern neighbors. Stitching requires an extra row and column of indices of the neighbor to be available to each tile. Thus, the actual tile size used is $512 \times 512$ with its last row and column being the first row and columns of the adjacent tiles. CPU sends separate stitch templates to effect correct stitching.

\subsection{Wireframe Rendering}

The rendering process can take two forms: software rendering (either in-house or by using licensed code) and hardware rendering (either directly using the hardware or via an API). The most common form used today, because of the high availability of graphics cards capable of real-time 3D graphics, is hardware rendering via an API such as Microsoft's Direct3D/DirectGraphics. To view the 3D DirectX generated wireframe mesh is rendered by the DEM data of the planet.

We present a scheme to render terrains, de-form them, edit them, and perform physics involving them at real-time rates. We use a representation that combines the fixed-size structure of geometry clip maps and the regularity of tiled blocks. The terrain is cached on the GPU using fixed-size rectangular blocks. The resolution of the blocks depends on the view and changes with height of the camera. A blocked, tiled, heightmap representation resides at the GPU cache at all times for fast rendering and real time modification. The cache is kept updated by sending data when needed for extent and resolution. 


\subsection{Stereoscopic Rendering}

The 3D viewing anaglyph is constructed using two images with an adequate look-angle, displayed in two colors (red and blue, or cyan), so that viewing with red-blue filter allows one image to be seen with the left eye (red glass) and the other to be seen with the right eye (blue glass). As long as the stereo separation is within limit of human viewing angle, the brain can perceive depth in the image. Anaglyph provides a valuable tool for visualizing the morphology of the Martian surface. This quick visualization tool assists in selecting scenes for labor intensive Digital Terrain Modeling.

The biggest hurdle in rendering detailed terrain data is the large data volume. The main limitation for the number of polygons to render was the bus bandwidth between CPU and GPU. Every highly detailed polygon mesh needed to be transferred over that bus. An additional limitation was the main memory of the graphics hardware. Even when it was managed to transfer the data, it couldn't be stored on the memory of the graphics card. To be able to render a high number of polygons on the graphics hardware, the way of how to render high polygon meshes needed to be rethought.

For the polygon mesh several discrete LODs were created. These discrete LODs had much less polygons. For rendering on the GPU the low poly mesh and the different generated LODs were used.

Stereo viewing provides added depth and realism to 3D views. This method requires a wear of special glasses with color filters over each eye. Best results we get, if used filters are of complementary colors. One can visualize 3D view in stereo by using red and blue stereo viewing glasses.

\section{RESULTS AND DISCUSSION}

Figure-2 shows developed application with zoom view of the mars surface. Figure- 3 shows the stepwise process of the algorithm.

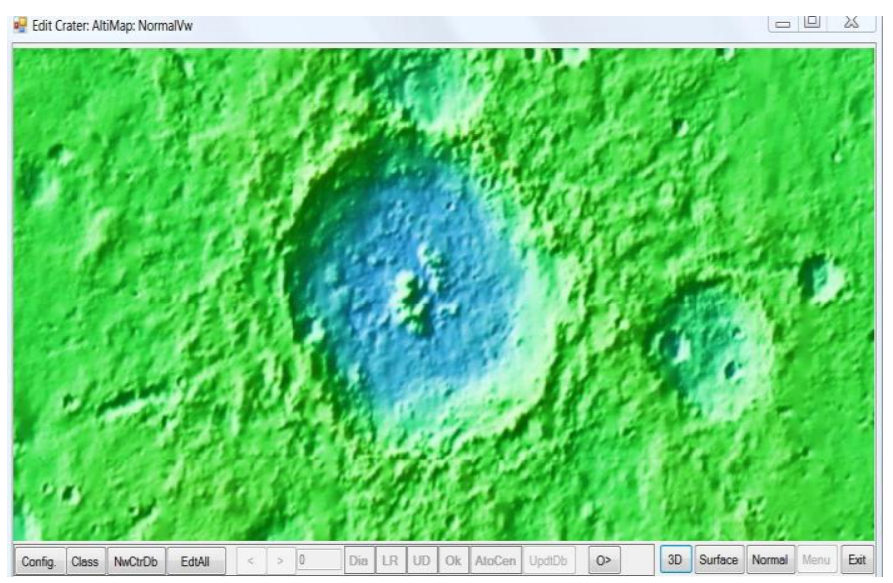

In figure 3, it shows the generated tiles of the Mars surface data and altitude data which is downloaded from the open source domain. After generating tiles of equal resolution, both the data tiles are stitched to create full surface map of the Mars.Using the DirectX technology wireframe mesh is generated with the DEM data of the Mars surface. Generated mesh is rendered with the Mars surface map data. So figure also shows the 3D visualization of the mars surface. To view in stereo 3D the map is projected at two different angles with two different color red and yellow for anaglyph view. Tool can also perform animated visualization of anaglyph 3D model.

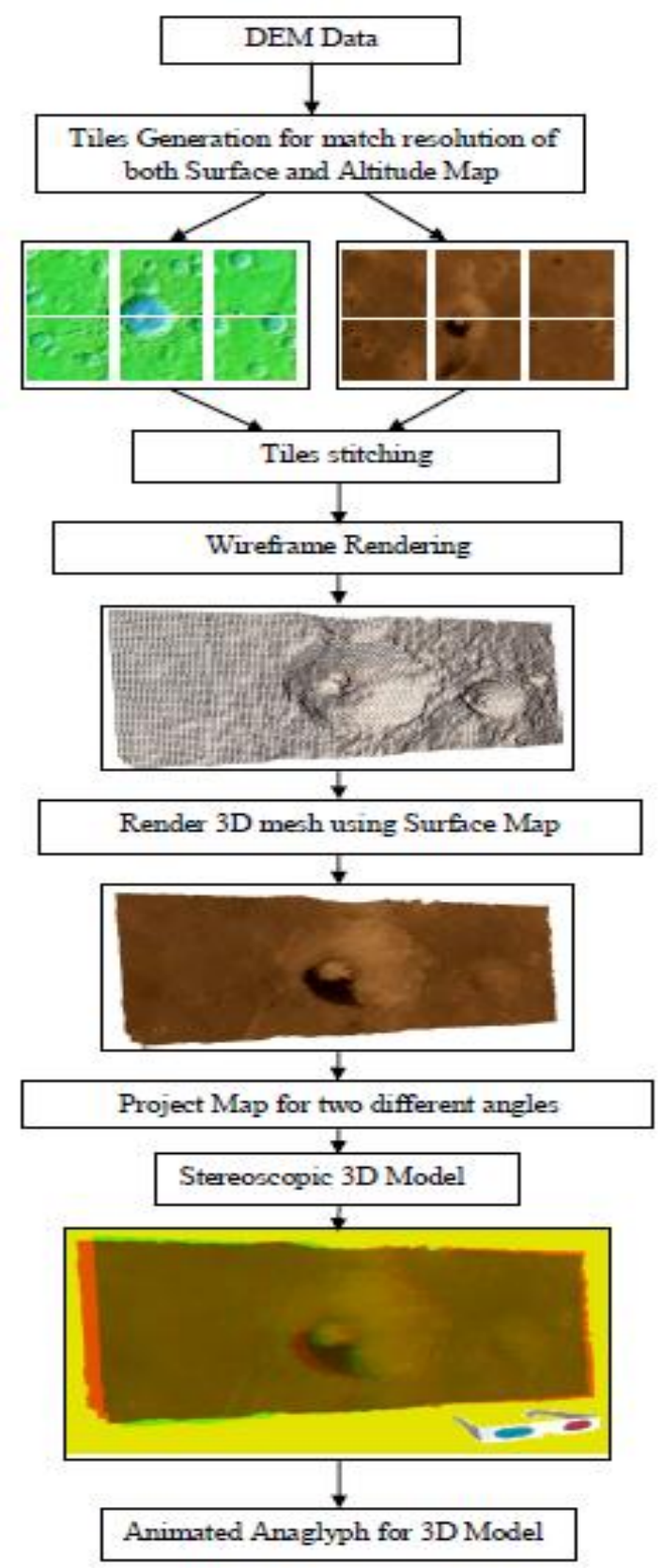

Fig 2: Mars surface zoomed view 
Fig 3: Shows the stepwise process of the creating animated anaglyph 3D model algorithm..

\section{CONCLUSIONS}

Uses include studies of relative and absolute surface age chronologies, erosion processes, Analysis of Planetary Surface and its formation Analysis of specific crater for scientific purpose, for safe landing site identification, mineralogical mapping of planet, development of 3D model from digital elevation map.

\section{ACKNOWLEDGEMENTS}

While bringing out this paper to its final form, we came across a number of people whose contributions in various ways helped our field of research and they deserve special thanks. It is a pleasure to convey my gratitude to all of them. I would like to take this opportunity to express my gratitude and heartiest thanks to Prof. R. S. Chhajed Head of the Department of Information Technology, for his support and constant encouragement.

\section{REFERENCES}

[1] Kiwon Lee, "A Prototype Implementation for 3D Animated Anaglyph Rendering of Multi-typed Urban Features using Standard OpenGL API", Korean Journal of Remote Sensing, Vol.23, No.5, 2007, pp.401 408.

[2] Krešimir Keresteš, "Making the Anaglyph Map"

[3] J. I. Simpson , J.R Kim b, J-P. Muller. 3D Crater Database Production On Mars By Automated Crater Detection And Data Fusion

[4] Tanvi Rajput. Satellite-Derived Digital Topographybased Crater Boundary Detection and Attribute Measurements by segmentation and Moments Measure Techniques.

[5] Y. Sawabe, T. Matsunaga, S.Rokugawa, Automated detection and classification of lunar craters using multiple approaches. Advances Space Research Volume 37, Issue 1, The Moon and Near-Earth Objects, pp.21-27, 2006

[6] Georg Held, Alias Abdul-Rahman, Siyka Zlatanova. Web 3D GIS for Urban Environments.

[7] http://www.mars.asu.edu/data/mola_color/ Mars Altitude

[8] www.celestiamotherlode.net/catalog/mars.php Mars Map

\section{BIOGRAPHIES}

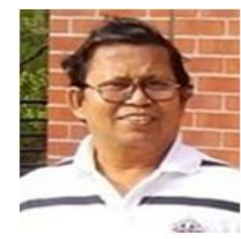

Himanshu S. Mazumdar acquired his Bachelor's degree in Engineering in the year 1968 and $\mathrm{PhD}$ in Computer Engineering in the year 2004. He has worked as Director, Research and Development, Defense Training \&
Technologies Champaign, IL, USA. Presently, he is working as Professor and Head of R\&D Center of Dharmsinh Desai Institute of Technology, Nadiad, India.

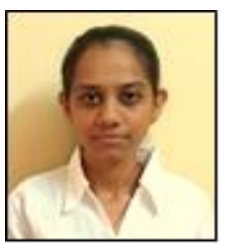
Divya J Nimavat received her Bachelor's degree in Computer Engineering in the year 2012 and M.Tech. in Information Technology in the year 2014. She has worked as Researcher at R\&D Center of Dharmsinh Desai Institute of Technology,

Nadiad, India.

Marichi Patel received his Bachelor and Master of Computer

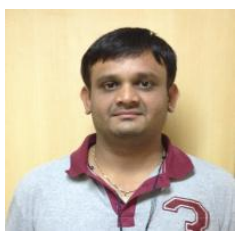
Application in the year 2009 and 2012 respectively. He has worked as Researcher and System Developer at R\&D Center of Dharmsinh Desai Institute of Technology, Nadiad, India. 